\title{
Metachronous gastric cancer risk after endoscopic resection of early gastric cancer and $H$. pylori status
}

\author{
Mototsugu Kato ${ }^{1}$
}

Received: 29 January 2019/Accepted: 5 February 2019/Published online: 15 February 2019

(C) Japanese Society of Gastroenterology 2019

Keywords Gastric cancer prevention · H. pylori eradication · Meta-analysis

Endoscopic resection for mucosal gastric cancer removes the tumor and surrounding mucosa. However, the remaining mucosa is also exposed to $H$. pylori, and possible carcinogens associated with metachronous gastric cancers may often develop in areas other than the resected primary tumor. Our first randomized study published in 2008 with an observation period of 3 years found that $H$. pylori eradication reduced the incidence of metachronous gastric cancer among patients who had undergone endoscopic resection of early gastric cancer [1]. Although a Korean randomized study with a similar protocol design contradicted our results [2], another Korean randomized study with a long-term follow-up confirmed that $H$. pylori eradication significantly inhibited occurrence of metachronous gastric carcinoma [3]. Furthermore, the preventive effect of gastric cancer development by $H$. pylori eradication was confirmed by five meta-analysis, including Xiao's manuscript entitled, "Helicobacter pylori status and risks of metachronous recurrence after endoscopic resection of early gastric cancer: a systematic review and meta-analysis" in this issue [4-8].

The mechanisms of the preventive effect of $H$. pylori eradication include slowing or stopping the growth of the latent cancers, and/or to shrink the cancers [9, 10]. Most latent cancers are too small to be detected by endoscopy,

Mototsugu Kato

mkato1957@gmail.com

1 National Hospital Organization, Hakodate Hospital, Hakodate, Japan some of which have grown large enough to be diagnosed during the follow-up. In addition, failure to detect synchronous cancer in the initial endoscopic screening complicates the number of metachronous cancers detected following endoscopic resection. To clarify the preventive effect of $H$. pylori eradication, a randomized study with a large sample and long-term observation period is required. Although a meta-analysis can alleviate the limitation in statistical power, further research is necessary to determine long-term progress. Choi IJ reported that metachronous gastric cancer developed in $7.2 \%(14 / 194)$ of patients in the eradication group and $13.4 \%(27 / 202)$ of patients in the placebo group during a median follow-up of 5.9 years (hazard ratio in the treatment group, 0.50; 95\% confidence interval, 0.26-0.94; $P=0.03$ ) [3].

Our previous multicenter, open-label, randomized controlled trial was based on 3 years of follow-up results regarding metachronous gastric cancer incidence following endoscopic resection of the primary cancer [1]. To evaluate the incidence of metachronous 10 years after entry of the first subject, 255 patients in the $H$. pylori eradication arm and 250 patients in the control arm attended at least one postrandomization were followed long term. The endpoint was development of a metachronous gastric cancer among the entire intention-to-treat (ITT) cohort using a logistic regression model containing the 544 subjects allocated and a modified ITT analysis with a proportional hazard model using 505 subjects with follow-up. The mean follow-up time was 4.96 years. Metachronous gastric cancer developed in 24 patients following eradication and in 43 controls. For the cohort of 544 subjects, the odds ratio was 0.51 (95\% confidence interval $0.30-0.87$ ), favoring eradication. In follow-up of 505 subjects, the hazard ratio was 0.54 (95\% CI 0.33-0.90). We concluded that preventive effect 
of $H$. pylori eradication for incidence of metachronous gastric cancer was sustained for a long term.

In Xiao's work, the incidence of metachronous gastric cancer was categorized according to three different $H$. pylori status: (1) H. pylori persistent infection: patients positive for $H$. pylori during follow-up regardless of eradication; (2) $H$. pylori eradication: patients infected with $H$. pylori at the time of endoscopic resection who received eradication therapy and subsequently confirmed as negative; and (3) $H$. pylori negative: $H$. pylori not detected during follow-up. Because gastric cancer occurring in mucosa without the history of $H$. pylori infection is rare, $H$. pylori negative cases include false negative or natural disappearance of $H$. pylori infection caused by severe atrophy and intestinal metaplasia. The risk of metachronous cancer in the vicinity of patients with $H$. pylori persistent infection or eradication has been unknown until this meta-analysis. Xiao's results showed no significant difference in recurrence risk between $H$. pylori negative and $H$. pylori persistent positive patients. These data suggest that carcinogenic effects of severe mucosal atrophic change including intestinal metaplasia cannot be ignored, despite the absence of $H$. pylori infection.

\section{References}

1. Fukase K, Kato M, Kikuchi S, et al. Effect of eradication of Helicobacter pylori on incidence of metachronous gastric carcinoma after endoscopic resection of early gastric cancer: an open-label, randomised controlled trial. Lancet. 2008;372:392-7.

2. Choi J, Kim SG, Yoon H, et al. Eradication of Helicobacter pylori after endoscopic resection of gastric tumors does not reduce incidence of metachronous gastric carcinoma. Clin Gastroenterol Hepatol. 2014;12:793-8003.

3. Choi IJ, Kook MC, Kim YI, et al. Helicobacter pylori therapy for the prevention of metachronous gastric cancer. N Engl J Med. 2018;378:1085-95.

4. Fuccio L, Zagari RM, Eusebi LH, et al. Meta-analysis: can Helicobacter pylori eradication treatment reduce the risk for gastric cancer? Ann Intern Med. 2009;151:121-8.

5. Ford AC, Forman D, Hunt RH, et al. Helicobacter pylori eradication therapy to prevent gastric cancer in healthy asymptomatic infected individuals: systematic review and meta-analysis of randomised controlled trials. BMJ. 2014;348:g3174.

6. Yoon SB, Park JM, Lim CH, et al. Effect of Helicobacter pylori eradication on metachronous gastric cancer after endoscopic resection of gastric tumors: a meta-analysis. Helicobacter. 2014;19:243-8.

7. Lee YC, Chiang TH, Chou CK, et al. Association between Helicobacter pylori eradication and gastric cancer incidence: a systematic review and meta-analysis. Gastroenterology. 2016;150:1113-24.

[8] Xiao S, Li S, Zhou L, et al. Helicobacter pylori status and risks of metachronous recurrence after endoscopic resection of early gastric cancer: a systematic review and meta-analysis. J Gastroenterol 2019. https://doi.org/10.1007/s00535-018-1513-8

9. Kato M, Asaka M, Ono S, et al. Eradication of Helicobacter pylori for primary gastric cancer and secondary gastric cancer after EMR. J Gastroenterol. 2007;42(Suppl 17):16-20.

10. Asaka M, Kato M, Graham DY. Prevention of gastric cancer by Helicobacter pylori eradication Intern Med. 2010;49(633):636.

Publisher's Note Springer Nature remains neutral with regard to jurisdictional claims in published maps and institutional affiliations. 